\title{
Recurrent hemoptysis in a 26-year-old woman with a ground-glass opacity lesion of the lung
}

\author{
Jong Ha Kim ${ }^{1}$, Sin-Youl Park ${ }^{2}$ \\ ${ }^{1}$ Department of Emergency Medicine, Kyungpook National University Chilgok-Hospital, Daegu, Korea \\ ${ }^{2}$ Department of Emergency Medicine, Yeungnam University College of Medicine, Daegu, Korea
}

Received: April 5, 2019

Revised: August 14, 2019

Accepted: August 23, 2019

Corresponding author:

Sin-Youl Park

Department of Emergency

Medicine, Yeungnam University

College of Medicine, 170

Hyeonchung-ro, Nam-gu, Daegu

42415, Korea

Tel: $+82-53-620-3626$

Fax: +82-53-623-8030

E-mail:dryuri@ynu.ac.kr
Hemoptysis is a major reason for emergency department (ED) visits. Catamenial hemoptysis (CH), a rare condition of thoracic endometriosis, can cause recurrent hemoptysis but is difficult to diagnose in the ED due to the scarcity of cases and nonspecific clinical findings. We report a case of a 26-year-old woman who presented to the ED with recurrent hemoptysis since 2 years without a definite cause. Her vital signs and blood test findings were unremarkable. Chest computed tomography (CT) did not show any specific lesions other than a non-specific ground-glass opacity pattern in her right lung. She was on day 4 of her menstrual cycle and her hemoptysis frequently occurred during menstruation. Although there was no histological confirmation, based on her history of hemoptysis during menstruation and no other cause of the hemoptysis, the patient was tentatively diagnosed with $\mathrm{CH}$ and was administered gonadotropin-releasing hormone. She had no recurrence of hemoptysis for 3 months. While $\mathrm{CH}$ is difficult to diagnose in the ED, the patient's recurrent hemoptysis related to menstruation was a clue to the presence of $\mathrm{CH}$. Therefore, physicians should determine the relationship between hemoptysis and menstruation for women of childbearing age presenting with repeated hemoptysis without a definite cause.

Keywords: Emergency; Endometriosis; Hemoptysis; Menstrual cycle; Menstruation

\section{Introduction}

Hemoptysis is a common respiratory symptom occurring in 7\%$15 \%$ of patients with respiratory disease [1]. Pulmonary tuberculosis, bronchiectasis, lung cancer, and bronchitis are the most common causes of hemoptysis. In addition, while approximately 40 other conditions can cause hemoptysis, the cause cannot be identified in about $30 \%$ of cases [2]. Massive hemoptysis, defined as bleeding of more than $100 \mathrm{~mL}$ at one time or more than 400 $600 \mathrm{~mL}$ in 24 hours, is associated with high mortality but occurs in only $1.5 \%$ of cases. Most hemoptysis cases do not present a life-threatening situation and usually resolve within 24 hours. However, hemoptysis is a major cause of emergency department
(ED) visits because its occurrence is anxiety-inducing for both patients and caregivers [3]. Because recurrent hemoptysis lowers patient quality of life and causes anxiety, ED visits increase in patients searching for the cause of their hemoptysis. Here, we present a case of a young woman who presented with recurrent hemoptysis for 2 years without a definite cause.

\section{Case}

A 26-year-old woman visited our ED for 2 days with recurrent hemoptysis. The woman produced blood-tinged sputum and the frequency of hemoptysis had increased gradually over 2 days. Her first hemoptysis event had occurred 2 years prior. The hemoptysis

Copyright(C) 2020 Yeungnam University College of Medicine

This is an Open Access article distributed under the terms of the Creative Commons Attribution Non-Commercial License (http://creativecommons.org/licenses/by-nc/4.0/) which permits unrestricted non-commercial use, distribution, and reproduction in any medium, provided the original work is properly cited. 
stopped without any special treatment but had recurred every 2-4 months. When hemoptysis had recurred 5 months prior, the patient had again visited the hospital. While her chest radiograph was unremarkable, chest computed tomography (CT) showed a lesion with a focal ground-glass opacity (GGO) pattern in her right lung (Fig. 1A). No abnormal findings were found in other examinations, including bronchoscopy. Her tuberculin test was negative. The hemoptysis improved after 2 days and the GGO-pattern lesion disappeared on follow-up CT. She had a 2 pack-year smoking history. The patient had no other known disease or family history. She was married but had never given birth. There was no history of pelvic surgery. Her menarche began at 14 years and her menstrual period was regular but with mild dysmenorrhea.

On presentation, the patient's blood pressure was $124 / 83$ $\mathrm{mmHg}$, pulse rate was 76 beats/min, oxygen saturation was $98 \%$, and she was afebrile. Her lung auscultation was clear. The laboratory results included a hemoglobin concentration of $10.9 \mathrm{~g} / \mathrm{dL}$ and platelet count of $373 \mathrm{~K} / \mu \mathrm{L}$. Her prothrombin/activated partial thromboplastin times were normal, at 13.4/30.8 seconds. Serum profiles including blood chemistry, electrolyte, and liver and renal function tests were normal. The serum levels of tumor markers such as cancer antigen 125 (CA 125) were not evaluated. Her urine was red in color. Chest radiographs were unremarkable. CT chest scans showed a focal GGO pattern in the right lower lung field in the same location as the lesion observed 5 months prior (Fig. 1B). While investigating the cause of her red-colored urine, we learned that the patient was on day 4 of her menstrual cycle and that her hemoptysis frequently occurred during men- struation. Based on her history of frequent hemoptysis during menstruation with no other cause of hemoptysis, she was tentatively diagnosed with catamenial hemoptysis $(\mathrm{CH})$. The patient was referred to a gynecologist for pelvic examinations, with no remarkable findings from ultrasonography or pelvic examination. Video-assisted thoracoscopic surgery (VATS) was planned for histological confirmation. However, she was reluctant to agree to surgical resection for diagnosis. The gynecologist administered gonadotropin-releasing hormone $(\mathrm{GnRH}, 3.75 \mathrm{mg}$ Leuplin DPS $^{\circledR}$ ) subcutaneously for control of her recurrent hemoptysis. She visited the gynecological clinic and additionally received GnRH injection once monthly for 3 months, during which time there was no recurrence of hemoptysis.

\section{Discussion}

Endometriosis is defined as the presence of endometrial glands and stroma outside the uterine cavity. Endometriosis is estimated to affect $3 \%-10 \%$ of fertile and $2 \%-5 \%$ of post-menopausal women. It is usually confined to the pelvis and rarely occurs in extra-pelvic areas. Thoracic endometriosis syndrome (TES) refers to the various clinical and radiological manifestations associated with the deposition of endometrial tissue in the lung parenchyma, pleura, diaphragm, and/or tracheobronchial tree. The incidence of TES is very rare. Nearly all cases have been reported through case reports and small retrospective series $[4,5]$. The four main types of TES are catamenial pneumothorax, catamenial hemothorax, $\mathrm{CH}$, and lung nodules. Of these, $\mathrm{CH}$ is an extremely rare manifestation, occurring in $7 \%$ of all TES cases [6].
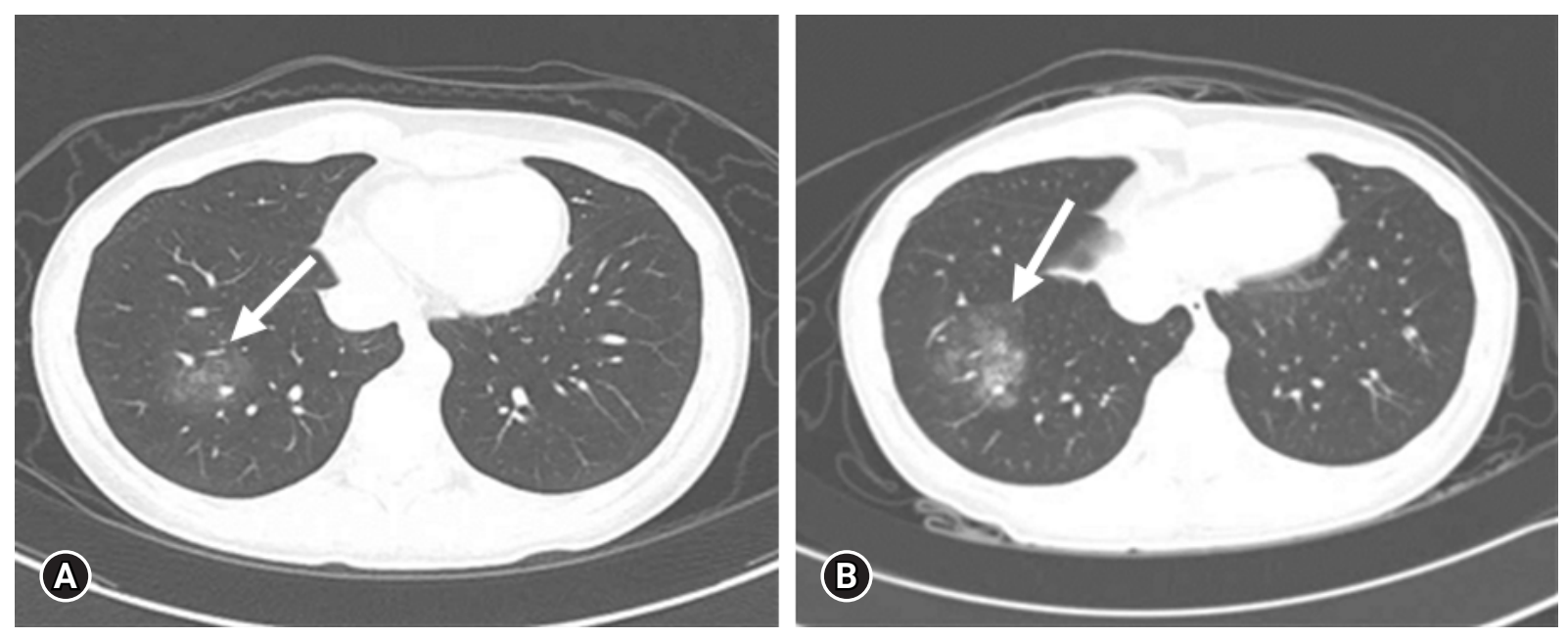

Fig. 1. Chest CT scan performed during menstruation. (A) A GGO lesion (arrow) is visible in the right lower lung field on chest CT performed 5 months prior. (B) A similar GGO lesion (arrow) is also visible in the same position in chest CT performed in the emergency department for hemoptysis. CT, computed tomography; GGO, ground-glass opacity. 
$\mathrm{CH}$ was first reported by Lattes et al. in 1956 and approximately 74 cases have been reported in the English literature since that time [7]. Unlike other forms of TES, pleuritic chest pain is not a common clinical presentation and symptoms may not occur with every menstrual cycle [8]. Fatal hemoptysis from $\mathrm{CH}$ has not yet been reported but it may cause life-threatening asphyxiation. Recent studies have reported massive hemoptysis requiring bronchial embolization in $\mathrm{CH}[9,10]$. In addition, recurrent hemoptysis by $\mathrm{CH}$ can reduce the quality of life of patients due to the associated respiratory symptoms and anxiety. Therefore, early recognition of $\mathrm{CH}$ has significance for patients. However, $\mathrm{CH}$ is not easy to diagnose because of the limited number of cases and its nonspecific physical examination, laboratory, and imaging findings. Auscultation is generally unremarkable and chest radiography and CT findings are nonspecific. Increased serum CA 125 levels have been reported, but only in patients with concurrent pelvic endometriosis [11]. Histologically, pulmonary endometriosis can be diagnosed when endometrial tissue is found in the lung parenchyma. Because most pathological lesions are located around the lung parenchyma, the role of bronchoscopy in diagnosis is limited [12]. Currently, VATS, which can directly observe the lung parenchyma, is the preferred method for histologic diagnosis; however, this method is invasive and can lead to complications [8]. $\mathrm{CH}$ is diagnosed by identification of the characteristic cyclic hemoptysis associated with menstruation after excluding other pulmonary causes of hemoptysis, for which treatment can be started on that basis [3]. Chest CT scans in $\mathrm{CH}$ often show nonspecific findings such as ill- or well-defined opacities, nodules, thin-walled cavities, bullous formations, and GGO patterns $[13,14]$. Although the results are nonspecific, a comparison of serial CT scans not only helps in the detection of $\mathrm{CH}$ but also in determining the underlying causes of the hemoptysis $[9,15]$. Pelvic endometriosis may help clinicians to suspect $\mathrm{CH}$; however, TES is not always accompanied by pelvic endometriosis. While $50 \%-85 \%$ of women diagnosed with TES have concomitant pelvic endometriosis, the incidence of TES in women with pelvic endometriosis is unknown [16].

$\mathrm{CH}$ can be treated medically or surgically; however, there are no clear treatment guidelines. Hormone therapy, including $\mathrm{GnRH}$, oral contraceptives, progesterone drugs, and danazol, is commonly used for the treatment of $\mathrm{CH}$. Our patient had no hemoptysis recurrence for 3 months with GnRH; however, longterm follow-up was not performed. With medical treatment alone, hemoptysis in $\mathrm{CH}$ recurs in up to $50 \%$ of patients [16]. Considering the pathogenesis of TES, inhibiting estrogen stimulation by removing endometrial tissue is reasonable and recent studies support the efficacy of combined surgical and medical therapy $[8,17]$

In conclusion, despite recent advances in diagnostic techniques,
$\mathrm{CH}$ is not easy to diagnose in the ED due to the scarcity of cases and its nonspecific examination results. However, recurrent hemoptysis due to $\mathrm{CH}$ causes not only patient anxiety and discomfort but may also cause asphyxia or massive hemoptysis. When ruling out the common causes of hemoptysis, identification of the relationship between hemoptysis and menstruation is helpful for the diagnosis of $\mathrm{CH}$. Therefore, physicians should assess this relationship in women of childbearing age presenting with repeated hemoptysis without a definite cause.

\section{Acknowledgments}

\section{Conflicts of interest}

No potential conflict of interest relevant to this article was reported.

\section{ORCID}

Jong Ha Kim, https://orcid.org/0000-0003-4131-5992

Sin-Youl Park, https://orcid.org/0000-0003-4005-1956

\section{References}

1. Garzon AA, Cerruti MM, Golding ME. Exsanguinating hemoptysis. J Thorac Cardiovasc Surg 1982;84:829-33.

2. Han CD, Kim YJ, Lee YS, Park JY, Jung TH. Evaluation of patients with hemoptysis and a normal chest roentgenogram. Tuberc Respir Dis 1995;42:42-9.

3. Jean-Baptiste E. Clinical assessment and management of massive hemoptysis. Crit Care Med 2000;28:1642-7.

4. Vinatier D, Orazi G, Cosson M, Dufour P. Theories of endometriosis. Eur J Obstet Gynecol Reprod Biol 2001;96:21-34.

5. Alifano M, Trisolini R, Cancellieri A, Regnard JF. Thoracic endometriosis: current knowledge. Ann Thorac Surg 2006;81:761-9.

6. Joseph J, Sahn SA. Thoracic endometriosis syndrome: new observations from an analysis of 110 cases. Am J Med 1996;100: 164-70.

7. Lattes R, Shepard F, Tovell H, Wylie R. A clinical and pathologic study of endometriosis of the lung. Surg Gynecol Obstet 1956;103:552-8.

8. Azizad-Pinto P, Clarke D. Thoracic endometriosis syndrome: case report and review of the literature. Perm J 2014;18:61-5.

9. Kim CJ, Nam HS, Lee CY, Yum HK, Yang SH, Seo KH, et al. Catamenial hemoptysis: a nationwide analysis in Korea. Respiration 2010;79:296-301.

10. Shin SP, Park CY, Song JH, Kim HM, Min D, Lee SH, et al. A case of catamenial hemoptysis treated by bronchial artery em- 
bolization. Tuberc Respir Dis 2014;76:233-6.

11. Hwang SM, Lee CW, Lee BS, Park JH. Clinical features of thoracic endometriosis: a single center analysis. Obstet Gynecol Sci 2015;58:223-31.

12. Hope-Gill B, Prathibha BV. Catamenial haemoptysis and clomiphene citrate therapy. Thorax 2003;58:89-90.

13. Rousset P, Rousset-Jablonski C, Alifano M, Mansuet-Lupo A, Buy JN, Revel MP. Thoracic endometriosis syndrome: CT and MRI features. Clin Radiol 2014;69:323-30.

14. Elliot DL, Barker AF, Dixon LM. Catamenial hemoptysis. New methods of diagnosis and therapy. Chest 1985;87:687-8.

15. Jang HI, Kim SE, Kim TJ, Lee YY, Choi CH, Lee JW, et al. Catamenial hemoptysis accompanied by subcutaneous endometriosis treated with combination therapy. Obstet Gynecol Sci 2017;60:236-9.

16. Channabasavaiah AD, Joseph JV. Thoracic endometriosis: revisiting the association between clinical presentation and thoracic pathology based on thoracoscopic findings in 110 patients. Medicine (Baltimore) 2010;89:183-8.

17. Alifano M, Roth T, Broët SC, Schussler O, Magdeleinat P, Regnard JF. Catamenial pneumothorax: a prospective study. Chest 2003;124:1004-8. 